\title{
Research on the Decision Mechanism of University-Enterprise Collaborative Innovation Based on Quantum Cognition
}

\author{
Sheng-Yuan Wang $\mathbb{D}^{1},{ }^{1}$ Wan-Ming Chen, ${ }^{2}$ Ying Liu, ${ }^{1}$ and Xiao-Lan Wu ${ }^{1}$ \\ ${ }^{1}$ Nanjing Xiao Zhuang University, Nanjing, Jiangsu, China \\ ${ }^{2}$ College of Economics and Management, Nanjing University of Aeronautics and Astronautics, Nanjing, Jiangsu, China
}

Correspondence should be addressed to Sheng-Yuan Wang; 56439976@qq.com

Received 10 February 2021; Revised 30 July 2021; Accepted 21 August 2021; Published 26 August 2021

Academic Editor: Harish Garg

Copyright $\odot 2021$ Sheng-Yuan Wang et al. This is an open access article distributed under the Creative Commons Attribution License, which permits unrestricted use, distribution, and reproduction in any medium, provided the original work is properly cited.

\begin{abstract}
The relationship between quantum cognition and decision-making is an important field in the direction of human behavior research. The uncertain and entangled state of quantum cognition has largely explained the decision-making process with limited information and ambiguity. University-enterprise collaborative innovation, a systematic project of joint action by both parties, aims to integrate the resources of enterprises and universities and enhance their innovation capabilities. As the main decisionmaking parties in collaborative innovation decision-making, the university and enterprise have a state of uncertainty and entanglement in their cognitive preferences before the final decision is formed. A university-enterprise collaborative innovation decision-making model should be constructed from the perspective of enterprise decision-making, taking different innovation schemes as the final choice, and the method of quantum probability theory can be used to perform quantum cognition on the independent and joint decision-making models in the university-enterprise collaborative innovation decision-making. Research shows that the internal mechanism of university-enterprise collaborative decision-making is based on its quantum attributes, university-enterprise collaborative decision-making is more inclined to joint decision-making, joint university-enterprise collaborative decision-making is more inclined to quantum cognition incompatibility expression, and the opinions of innovative members will have sequential effects and interference effects on decision makers.
\end{abstract}

\section{Introduction}

In recent years, quantum theory has been used to study human behavior science. Quantum theory is a set of scientific theories confirmed with successful experience. It is an indispensable theoretical knowledge for the research and application of "natural subjects" and has become an important focus of cognitive science. Through axiomatic abstract theories, quantum theory is used to solve similar problems in nonquantum systems. Quantum game and quantum strategy, proposed based on the game and strategy research of quantum theory, have solved the Nash equilibrium [1] in "prisoner's dilemma," and then the concept of quantum game has gradually expanded to related fields of economics, and a new quantum model research on relationships between stock prices and equity [2], the quantum mechanical model of stock returns [3], and the quantum dynamics of the stock market $[4,5]$ have appeared. The development of quantum games and the application of quantum theory in economics have further improved the mathematical axioms of quantum theory, enabling it to be gradually applied to human behavior decision-making. Quantum cognition is a new research project that starts from quantum theory and uses mathematical principles as framework to explain human cognition, including judgment and decision-making, concepts, reasoning, memory, and perception. Quantum theory is used as a new conceptual framework and a set of formulated tools to explain the puzzling research results in empirical research in psychology [6].

In 1995, Bruza and Gabora discovered for the first time that human judgment and decision-making have quantum 
properties [7]. The human decision-making process can be described by quantum theory [8], which can explain the intuition and bias in decision-making and judgment [9-11]. The link between the basic concepts of quantum physics and psychology is proposed [12]. The framing effect and cognitive dissonance can be explained well by the geometric properties (the Heber space describes the state of events) in quantum theory [13]. It is proved that even if the brain is classically probabilistic, the observed cognitive behaviors and processes may be better modeled by quantum probability $[14,15]$. The experiment on the concept of "Pet-Fish" reveals the quantum in cognition structure, that is, why and how concepts are entangled [16]. The experiment that uses geometric properties in quantum theory to study and explain the "Gore-Clinton problem" shows that the context effect of the problem sequence reveals the quantum nature of human judgment [17], which is a key successful case of applying quantum theory in human cognition $[18,19]$. The Fock space developed from the perspective of quantum theory can successfully model different cognitive experiments, including the modeling of human decision-making [20]. "Narrow and wide face experiment" and "feminist experiment" explained the effect of measurement order in psychology and proposed a priori equation for the order effect of quantum cognition, which further proved the usability of quantum cognition [21]. In a task of identifying the direction of movement, an interference effect was found in human judgment [22]. Compared with the Markov model widely used in cognitive psychology, the interference effect produced by quantum probability can better explain the deviation in the decision-making process.

So far, the rapid development of quantum cognition has mainly focused on six aspects: decision-making process, fuzzy perception, semantic network, probability judgment, order effect of measurement cognition, and memory. The effective application of quantum theory in the field of human cognition has been proved $[23,24]$. Human cognition and decision-making "behavior" have quantum characteristics [25]. Quantum research on organizational decision-making has proved that there are also quantum attributes in organizational decisionmaking [26]. Quantum cognitive models are universally present in individual cognitive behaviors and various behavioral activities in human society. Cognitive decision-making is also a quantum process. The quantum decision-making process mainly uses quantum probability. Quantum cognition, quantum probability, and quantum decision-making are increasingly applied to research fields such as management and economics.

Innovation activity is a systematic engineering problem as it is difficult for an organization to have all the resources needed for innovation. In the specific regional innovation environment, where innovation resources are limited, the material flow and talent flow among different innovation groups are interrelated and transmitted, forming a dynamic evolution and symbiosis. The basic problem to be solved by the theoretical research and social practice in the field of economic management is how to optimize the allocation of resources [27-29].
One of the key measures for collaborative innovation of industry, university, and research is to strengthen the relationship between the two innovation subjects, namely, university and enterprise, which is the inevitable result of the choice of knowledge heterogeneity, public goods attribute and new system by the two subjects. From the perspective of cognitive psychology, behavioral misunderstandings and decision-making cognitive biases are caused by decision makers' decision-making judgments under the condition of "bounded rationality." Based on a completely rational assumption, these traditional research methods get the conclusion that has bypassed the universal "bounded rationality" and uncertainty. The rational decision theory is difficult to explain these deviating decision-making behaviors affected by psychological, environmental factors and the cognitive abilities of decision makers. There is also a certain degree of difficulty in the entanglement between the members' complex cognitive information in the collaborative innovation system. When decision makers receive the opinions of university-enterprise cooperative members, the superposition, entanglement, and uncertainty of their personal cognitions that cannot be completely rationally explained, how, based on the assumption of "bounded rationality," to explain these deviations that cannot be explained by traditional rational decisions and how these deviations affect university-enterprise collaborative innovation decisions have become the focus of research. The quantum decision-making method has been successfully applied in the field of multiobjective decision-making and multicriteria decision-making [30, 31]. Having referred to the relevant research, this paper is motivated to find a decision-making method that can solve the above cognitive bias problems.

The contents of this paper are as follows. Based on the framework of quantum cognitive theory and quantum probability theory, through literature analysis, a universityenterprise collaboration innovative decision-making model is to be established combining cognitive decision-making theory, related theories on university-enterprise collaborative consumer behavior, and the research conclusions of university-enterprise collaborative innovation behavior. The university-enterprise collaborative purchasing decisionmaking process is taken as a unified and opposite whole to study the university-enterprise collaborative decisionmaking. Its unity is manifested in that the final decisionmaking result reflects the cognition and preference of university-enterprise collaborative members, and its opposition is reflected in that the cognition and preference status of university and enterprise are relatively independent in the transition from innovation intention to innovation decisionmaking. The mutual influence between the preference states forms the final decision, and the interaction produced by the entanglement between the preferences is the focus of research. The quantum probability interpretation of the university-enterprise joint decision-making model is further analyzed, and the sequential effects and interference effects that appear in the joint model are analyzed.

The highlight of this study is being able to clarify the usability of quantum cognitive theory in university- 
enterprise collaborative decision-making. Compared with traditional methods which are based on the assumption of "rational man" that eliminates uncertainty and entanglement, quantum methods have the most direct recognition of decision makers. The interpretation of state changes enriches the research on the internal causes of university-enterprise collaborative decision-making and also enriches the research on quantum cognition in the field of innovative decisionmaking.

\section{Materials and Methods}

Based on the simple model proposed by BuseEeyer and Bruza [32], we suppose that a pair of university-enterprise collaborative innovation partners is considering an innovation cooperation project for the needs of university-enterprise collaborative innovation. After extensive research, demonstration, comparison, and selection, they decide to choose one of $N$ innovative cooperation projects and put it into practice. $N=\left\{x_{1}, x_{2}, \ldots, x_{n}\right\}$, and the set represents different innovation cooperation projects.

This university-enterprise collaborative innovation decision is to be analyzed from the perspective of enterprise decision-making. The enterprise itself is also a decision maker. There is a single categorical variable "E." The enterprise chooses an innovation cooperation project; that is, $E$ chooses an innovation cooperation project, which may produce $n$ possibilities of results.

The variable " $X$ " is a measure of corporate preferences, which will produce $n$ detailed and mutually exclusive results: $E=\left\{E x_{1}, E x_{2}, \ldots, E x_{n}\right\}$. Innovation decision-making is the final joint decision-making when the enterprise chooses one of the innovation cooperation projects among the $N$ projects after fully considering the preferences of both the university and the enterprise. The preferences of the enterprise and of the university for the innovation cooperation project exist simultaneously and influence the final decision of the enterprise. The superposition of these two uncertain states in the quantum model will show a two category variable situation; that is, in addition to the enterprise's own point of view, there is also a preference of the university that the enterprise thinks. The second classification variable measures the preference of the university that the enterprise thinks is " $U$," which is expressed as $y_{i}$, different from the enterprise's preference. This can also produce $n$ detailed and mutually exclusive results: $U=\left\{U y_{1}, U y_{2}, \ldots, U y_{n}\right\}$ [13].

Geometric method and path map method are two modeling methods commonly used in quantum cognitive theory. In this paper, the geometric modeling method is used to model the hypothesis in the research of university-enterprise collaborative purchase decision. The vector space can be used to simulate the decision-making preference of the enterprise and that of the university perceived by the enterprise. They are expressed by the values of two classification variables $E$ and $U$, respectively.

The multidimensional space constructed with geometric method is abstract, and the coordinate axes of a basic vector are orthogonal and are of unit length. The initial state of university-enterprise collaborative decision maker's preference can be at any point in multidimensional space crossed by these coordinate axes. Using the Bra-ket notation, the base vector is called a "right vector." The base vector of the $x_{1}$-axis is represented by the symbol $\left|x_{1}\right\rangle$. Adding a "number" before this span indicates that any point on the $x_{1}$-axis can be represented by $a\left|x_{1}\right\rangle$, where $a$ is a scalar.

Similarly, enterprises believe that the preference of universities can be expressed by $\left|U y_{1}\right\rangle,\left|U y_{2}\right\rangle, \ldots,\left|U y_{n}\right\rangle$, respectively. According to quantum theory, the probability of an independent event is obtained by projecting the state vector onto the axis representing the event and then squaring the magnitude of the projection.

The state system $S$ of the enterprises' selection of innovation cooperation projects indicates the initial preference state of enterprises for these innovation cooperation projects and is the starting point of enterprise innovation cooperation project decision-making. It can be expressed by each event and its corresponding amplitude:

$$
|S\rangle=\psi(x)=\psi_{1}\left|x_{1}\right\rangle+\psi_{2}\left|x_{2}\right\rangle+\cdots+\psi_{n}\left|x_{n}\right\rangle .
$$

These basic events are mutually exclusive and the basic vectors corresponding to the basic results are orthogonal.

The vector $\left|x_{1}\right\rangle,\left|x_{2}\right\rangle, \ldots,\left|x_{n}\right\rangle$ is based on the column vector corresponding to the coordinate axis of the basic vector $\left\{\left|x_{1}\right\rangle,\left|x_{2}\right\rangle, \ldots,\left|x_{n}\right\rangle\right\}$ as shown in equation (1), and the vectors are orthogonal and mutually exclusive and have a unit length of 1 .

$$
\begin{aligned}
\left|x_{1}\right\rangle & \longrightarrow\left[\begin{array}{llll}
1 & 0 & \ldots & 0
\end{array}\right]^{T} \\
\left|x_{2}\right\rangle & \longrightarrow\left[\begin{array}{llll}
0 & 1 & \ldots & 0
\end{array}\right]^{T} \\
\ldots & \\
\left|x_{n}\right\rangle & \longrightarrow\left[\begin{array}{llll}
0 & 0 & \ldots & 1
\end{array}\right]^{T} \\
|S\rangle & \longrightarrow\left[\begin{array}{llll}
\psi_{1} & \psi_{2} & \ldots & \psi_{n}
\end{array}\right]^{T}=\psi .
\end{aligned}
$$

The value of $\psi$ is an initial preference obtained from the cognition of these innovation cooperation projects. This preference is in a state of constant change and uncertainty and is difficult to measure. In quantum theory, state is a unit length vector in $\mathrm{N}$-dimensional vector space, which is symbolized to map events to probability, and the mapping from state to probability is indirect. The state is projected to the subspace corresponding to the event, and the square length of the projection is equal to the event probability. The probability of enterprise that chooses innovation cooperation project is the square length of projection from state vector to event. The mapping from state to probability is nonlinear. It involves the square of amplitude, the projection at zero point is mapped to zero probability, and the projection in the whole vector space is mapped to the probability that is equal to 1 .

\subsection{Decision Event Probability}

2.1.1. Probability of Single Events. The coordinates assigned to each base vector can be obtained by the inner product of the basis vector and the state vector. Based on the basic 
coordinates $\left\{\left|x_{1}\right\rangle,\left|x_{2}\right\rangle, \ldots,\left|x_{n}\right\rangle\right\}$, the inner product of $\left|x_{1}\right\rangle$ and $|S\rangle$ can be calculated by the following matrix formula:

$$
\left\langle x_{1} \mid S\right\rangle=\left[\begin{array}{llll}
1 & 0 & \ldots & 0
\end{array}\right] \cdot\left[\begin{array}{c}
\psi_{1} \\
\psi_{2} \\
\ldots \\
\psi_{n}
\end{array}\right]=\psi_{1} \text {. }
$$

The inner product $\langle X \mid S\rangle$ represents the transition amplitude from state $|S\rangle$ to state $|X\rangle$ proposed by Feynman. $\left\langle x_{1} \mid S\right\rangle$ is the transition amplitude from the initial state $S$ to the enterprise's decision to select state $x_{1}$. Under the decision-making of joint models and independent models, there is a probability difference in the choice of an innovation cooperation project. Unlike the classical probability theory, which uses set representation to model events, quantum theory models events as quantum space instead of sets. The hypothesis of quantum theory is based on a set of basis vectors. An enterprise chooses an innovation project as an event. In the model, there are $N$ kinds of innovation cooperation projects to choose from, and there are $N$ events that enterprises can make decisions on. It is expressed by vector as follows: $\left\{\left|x_{1}\right\rangle,\left|x_{2}\right\rangle, \ldots,\left|x_{n}\right\rangle\right\} .\left|x_{1}\right\rangle$ means that the enterprise chooses innovation cooperation project $x_{1}$. The probability of selecting innovation cooperation project $x_{1}$ is $p\left(X=x_{i}\right)=\left|\psi_{i}\right|^{2}=\psi_{i} * \psi_{i}, \quad \sum_{i=1}^{n}\left|\psi_{i}\right|^{2}=1 . \quad \psi_{i}$ is the state range of enterprises' preference for innovation cooperation project $x_{1}$. The amplitude can be expressed by complex wave function: $\psi_{i}=a_{i}+b_{i} i$. The same probability is obtained by state vector projection by projecting the state vector onto the basis vector corresponding to $x_{1}$, the length of the amplitude is $\left|x_{i}\right\rangle\left\langle x_{i} \mid S\right\rangle=\psi_{i} \cdot\left|x_{i}\right\rangle$. Square the amplitude length and we get the probability $\| \psi_{i} \cdot\left|x_{i}\right\rangle\left\|^{2}=\left|\psi_{i}\right|^{2} \cdot\right\|\left|x_{i}\right\rangle \|^{2}=|\psi i|^{2} \cdot 1$.

2.1.2. Probability of Other Possible Events. If $x_{1}, x_{2}, \ldots, x_{i}$ is the $i$-th item in the $N$ innovation cooperation projects, then the probability that the enterprise chooses the $i$-th innovation cooperation project is projected from the state $S$ to the square of the subspace length that the base vector $x_{1}, x_{2}, \ldots, x_{i}$ crosses. The probability of this event is obtained by projecting the state vector $S$ into this subspace. The projection equation is

$$
\left(\left|x_{1}\right\rangle\left\langle x_{1}|+| x_{2}\right\rangle\left\langle x_{2}|+\cdots+| x_{i}\right\rangle\left\langle x_{i}\right|\right)|S\rangle=\left|x_{1}\right\rangle\left\langle x_{1} \mid S\right\rangle+\left|x_{2}\right\rangle\left\langle x_{2} \mid S\right\rangle+\cdots+\left|x_{i}\right\rangle\left\langle x_{i} \mid S\right\rangle .
$$

The probability of this event is the square of the length of the event projection equation. $\left|x_{1}\right\rangle,\left|x_{2}\right\rangle, \ldots,\left|x_{i}\right\rangle$ is orthogonal to ensure that the sum of the squares of each length equals the square of the total length.

\subsection{Joint Decision-Making of University-Enterprise Preference} Judgment without Sequence. University-enterprise collaborative decision-making mainly adopts independent decision-making type and joint decision-making type. In independent decision-making, enterprises can make decisions independently without thinking about the preference state of universities. The probability of university-enterprise collaborative decision-making can be obtained by calculating the probability of a single classification variable. For the joint university-enterprise collaborative decision-making, enterprises have to think about the preference of universities, and there will be a second category variable, which leads to the problem of quantum compatibility.

In quantum theory, decision makers are allowed to have compatible and incompatible states for events. The most important difference between the preference states of both sides in the formation of collaborative joint decision-making between universities and enterprises is that the decision makers are in compatible or incompatible states.

When the enterprise can judge the preference states of the university and the enterprise at the same time, in quantum cognition, it is considered that they have sufficient information about each innovation cooperation project and have enough experience to judge the preference choice of the university and its own innovation cooperation project at the same time. When the first category variable $E$ and the second category variable $U$ can be considered at the same time, they belong to compatible representation, composing $N \times N$ different combinations. It is not important to consider the sequence of the two parties' preferences.

$$
\begin{gathered}
\left(E x_{1}, U y_{1}\right),\left(E x_{1}, U y_{2}\right), \ldots\left(E x_{1}, U y_{n}\right) \\
\left(E x_{1}, U y_{1}\right),\left(E x_{2}, U y_{2}\right), \ldots\left(E x_{2}, U y_{n}\right) \\
\ldots \\
\left(E x_{n}, U y_{1}\right),\left(E x_{n}, U y_{2}\right), \ldots\left(E x_{n}, U y_{n}\right)
\end{gathered}
$$

The initial preference state of enterprises in these combination events can be expressed as follows:

$$
\begin{aligned}
|S\rangle= & \psi_{x_{1} y_{1}} \cdot\left|x_{1} y_{1}\right\rangle+\psi_{x_{1} y_{2}} \cdot\left|x_{1} y_{2}\right\rangle+\cdots+\psi_{x_{1} y_{n}} \cdot\left|x_{1} y_{n}\right\rangle \\
& +\psi_{x_{2} y_{1}} \cdot\left|x_{2} y_{1}\right\rangle+\psi_{x_{2} y_{2}} \cdot\left|x_{2} y_{2}\right\rangle+\cdots+\psi_{x_{2} y_{n}} \cdot\left|x_{2} y_{n}\right\rangle \\
\cdots & \\
& +\psi_{x_{n} y_{1}} \cdot\left|x_{n} y_{1}\right\rangle+\psi_{x_{n} y_{2}} \cdot\left|x_{n} y_{2}\right\rangle+\cdots+\psi_{x_{n} y_{n}} \cdot\left|x_{n} y_{n}\right\rangle .
\end{aligned}
$$

The first letter represents the preference of enterprises, and the second letter represents the preference of universities. For example, the base vector $\left|x_{1} y_{1}\right\rangle$ represents the event "the enterprise prefers item 1 and the enterprise thinks that the university also prefers item 1 ," and there are $N \times N$ coordinates corresponding to each basic vector. The probability of $N \times N$ events is obtained by projecting the state $|S\rangle$ onto the corresponding basic vector.

The probability that enterprise prefers $x_{i}$ and their cooperative university prefers $x_{j}$ is $\| \psi_{x_{i} x_{j}} \cdot\left|x_{i} x_{j}\right\rangle \|^{2}=\left|\psi_{x_{i} x_{j}}\right|^{2}$. 
If the product $\alpha_{i} \cdot \beta_{j}$ of two coefficients can be separated from the joint coefficient $\psi_{x_{i} x_{j}}$ so that $\psi_{x_{i} x_{j}}=\alpha_{i} \cdot \beta_{j}$ for all pairs of $(i, j)$, then the state is considered separable; otherwise, the state is entangled.

Enterprises can consider the preferences of themselves and that of the universities at the same time, because these two variables can share a set of sample space to describe all events, and the two variables $E$ and $U$ are compatible. Also based on the combination of $N \times N$ events, the revision of the initial state of an enterprise can be divided into three steps: first, the initial state $|S\rangle$ is projected onto the subspace crossed by the basis vector $\left\{\left|x_{1} y_{j}\right\rangle,\left|x_{2} y_{j}\right\rangle, \ldots,\left|x_{n} y_{j}\right\rangle\right\}$, which is consistent with the fact that "the enterprise has known that the university prefers innovation cooperation projects $y_{j}$," and then the state projection $\psi_{x_{1} y_{j}}\left|x_{1} y_{j}\right\rangle+$ $\psi_{x_{2} y_{j}}\left|x_{2} y_{j}\right\rangle+\cdots+\psi_{x_{n} y_{j}}\left|x_{n} y_{j}\right\rangle$ is generated. Secondly, the square length of the projection is calculated; that is, the probability of the university's preference for $y_{j}$ is $p_{1}=\sum_{k=1}^{n}\left|x_{k} y_{j}\right|^{2}$. Finally, the modified state $\left|S_{R}\right\rangle$ is equal to the normalized projection, and the normalized state length is 1 .

$$
\left|S_{R}\right\rangle=\frac{\sum_{k=1}^{n}\left(\psi_{x_{k} y_{j}}\left|x_{k} y_{j}\right\rangle\right)}{\sqrt{\sum_{k=1}^{n}\left|x_{k} y_{j}\right|^{2}}} .
$$

From the revised state $\left|S_{R}\right\rangle$, it can be seen that the probability of the university choosing $y_{j}$ is equal to 1 , which is consistent with the fact that the university prefers $y_{j}$ innovation cooperation projects. The process of state revision is similar to the calculation of conditional probability in classical probability model, and it also needs to be standardized based on conditional events.

The revised state $\left|S_{R}\right\rangle$ is obtained through the state revision process, and the probability of enterprise preference for project $x_{i}$ is calculated by using the state $\left|S_{R}\right\rangle$. State $\left|S_{R}\right\rangle$ is projected onto the subspace covered by $\left\{\left|x_{i} y_{1}\right\rangle,\left|x_{i} y_{2}\right\rangle, \ldots,\left|x_{i} y_{n}\right\rangle\right\}$, and the probability $p_{2}=\left|\psi_{x_{i} y_{j}}\right|^{2} / \sum_{k=1}^{n}\left|x_{k} y_{j}\right|^{2}$ is obtained by squaring it. Under this sequence of consideration and preference, the probability that an enterprise finally chooses $x_{i}$ innovation cooperation project is equal to the product of $p_{1}$ and $p_{2}$ probabilities: $p_{1} p_{2}=\left|\psi_{x_{i} y_{j}}\right|^{2}$. If the preference of enterprises is evaluated first, and before considering the preference of colleges and universities, the probability $\left|\psi_{x_{i} y_{j}}\right|^{2}$ will be obtained exactly the same as in the previous analysis. When the preference variables of enterprises and universities are compatible, there is no entanglement state in the preference of enterprises and universities, and the sequence of consideration of enterprises has no influence on the final selection result. This conforms to the classical probability theory, in which the sequential effect is not involved. In compatible representation, the sequence of preference consideration is not important.

2.3. Joint Decision-Making with the Sequence of Preference Judgment between the University and Enterprise. The sequential effect of university-enterprise collaborative decision-making is whether the decision makers give priority to their own cognitive state or that of university-enterprise collaborative members. There are two sequential paths. As a decision maker, an enterprise first considers their own preference and then the university's preference, and the enterprise first considers the university's preference and then their own preference. The preference state of one side of the university-enterprise is determined before decisionmaking, and the final decision-making of the enterprise will be affected to a certain extent. It is necessary to project the initial state of the enterprise into a modified state, so as to ensure that the preference of the university that the enterprise thinks about conforms to the known definite information; that is, from the original two uncertain states to an uncertain state, the next is the analysis of this impact from a quantum perspective.

\subsubsection{University-Enterprise Collaborative Decision-Making} with Sequential Effect. Two variables are regarded as incompatible, and there are two vector bases to describe the two variables, respectively. The $\left\{\left|x_{1}\right\rangle,\left|x_{2}\right\rangle, \ldots,\left|x_{i}\right\rangle\right\}$ basis is used to show the selection of innovation cooperation projects from the perspective of enterprises, and a new basis $\left\{\left|y_{1}\right\rangle,\left|y_{2}\right\rangle, \ldots,\left|y_{i}\right\rangle\right\}$ is used in the same space to express the preference of enterprises for their universities.

(1) Variable Transformation Matrix: Unitary Matrix. The same state $|S\rangle$ can get different coordinates with different basic vectors. In this paper, the columns involved in the coordinate system of enterprise preference and university preference are sorted into $N \times N$ transform matrix $U_{E U}$. The transformation amplitude matrix must be unitary matrix; then, there is $U_{E U} \cdot U_{E U}^{\dagger}=I=U_{E U}^{\dagger} \cdot U_{E U}$, where $I$ is the identity matrix. According to the restriction requirements of quantum model, the sum of squares of each row or column must be 1 , which is the double randomness that geometric modeling method must satisfy.

$$
\begin{aligned}
U_{E U}= & {\left[\begin{array}{cccc}
\left\langle x_{1} \mid y_{1}\right\rangle & \left\langle x_{1} \mid y_{2}\right\rangle & \ldots & \left\langle x_{1} \mid y_{n}\right\rangle \\
\left\langle x_{2} \mid y_{1}\right\rangle & \left\langle x_{2} \mid y_{2}\right\rangle & \ldots & \left\langle x_{2} \mid y_{n}\right\rangle \\
\vdots & \vdots & \vdots & \vdots \\
\left\langle x_{n} \mid y_{1}\right\rangle & \left\langle x_{n} \mid y_{n}\right\rangle & \ldots & \left\langle x_{n} \mid y_{n}\right\rangle
\end{array}\right], } \\
U_{E U}^{\dagger} & =U_{U E}=\left[\begin{array}{cccc}
\left\langle y_{1} \mid x_{1}\right\rangle & \left\langle y_{1} \mid x_{2}\right\rangle & \ldots & \left\langle y_{1} \mid x_{n}\right\rangle \\
\left\langle y_{12} \mid x_{n}\right\rangle & \left\langle y_{2} \mid x_{2}\right\rangle & \ldots & \left\langle y_{2} \mid x_{n}\right\rangle \\
\vdots & \vdots & \vdots & \vdots \\
\left\langle y_{n} \mid x_{1}\right\rangle & \left\langle y_{n} \mid x_{2}\right\rangle & \ldots & \left\langle y_{n} \mid x_{n}\right\rangle
\end{array}\right] .
\end{aligned}
$$

Through the unitary matrix, the initial state $|S\rangle$ coordinate $\psi$ represented by the enterprise perspective basis vector $\left\{\left|x_{1}\right\rangle,\left|x_{2}\right\rangle, \ldots,\left|x_{i}\right\rangle\right\}$ can be transformed into the initial state $|S\rangle$ coordinate $\varphi$ represented by the basis vector $\left\{\left|y_{1}\right\rangle,\left|y_{2}\right\rangle, \ldots,\left|y_{i}\right\rangle\right\}$ of the university perspective. The value of $\varphi_{i}$ represents the university's preference, perceived by the enterprise, for the corresponding innovation cooperation projects: $|S\rangle \longrightarrow U_{E U}^{\dagger} . \Psi=\varphi$ or ${ }_{U}^{E U} \cdot \varphi=\Psi$. 
It also has a unit length: $\||S\rangle\left\|^{2}=\right\| \varphi \|^{2}=1 . \varphi$ and $\psi$ are coordinates that involve different basic vector axes in the same system of $|S\rangle$, where $|S\rangle$ is taken as abstract vector.

(2) Representation of Different Variables with the Same Basis Vector. Any point in quantum multidimensional space can be achieved by linear combination of $\left\{\left|y_{1}\right\rangle,\left|y_{2}\right\rangle, \ldots,\left|y_{n}\right\rangle\right\}$ basis vectors. Based on the unitary matrix $U_{E U}$, with respect to $\left\{\left|y_{1}\right\rangle,\left|y_{2}\right\rangle, \ldots,\left|y_{n}\right\rangle\right\}$, the $\left\{\left|x_{1}\right\rangle,\left|x_{2}\right\rangle, \ldots,\left|x_{n}\right\rangle\right\}$ vector can be described as follows:

$$
\left|x_{i}\right\rangle=\left\langle x_{i} \mid y_{1}\right\rangle\left|y_{1}\right\rangle+\left\langle x_{i} \mid y_{2}\right\rangle\left|y_{2}\right\rangle+\cdots+\left\langle x_{i} \mid y_{n}\right\rangle\left|y_{n}\right\rangle \text {. }
$$

Similarly, $\left\{\left|y_{1}\right\rangle,\left|y_{2}\right\rangle, \ldots,\left|y_{n}\right\rangle\right\}$ can be represented by a linear combination of $\left\{\left|x_{1}\right\rangle,\left|x_{2}\right\rangle, \ldots,\left|x_{n}\right\rangle\right\}$ :

$$
\left|y_{i}\right\rangle=\left\langle y_{i} \mid x_{1}\right\rangle\left|x_{1}\right\rangle+\left\langle y_{i} \mid x_{2}\right\rangle\left|x_{2}\right\rangle+\cdots+\left\langle y_{i} \mid x_{n}\right\rangle\left|x_{n}\right\rangle \text {. }
$$

(3) Sequential Effect in Joint Decision-Making. When an enterprise first considers the university's preference $\left|y_{j}\right\rangle$ and then their own preference $\left|x_{i}\right\rangle$, the sequence of events path is expressed as follows: $|S\rangle \longrightarrow\left|y_{j}\right\rangle \longrightarrow\left|x_{i}\right\rangle$, using all calculated coordinates based on $\left\{\left|x_{1}\right\rangle,\left|x_{2}\right\rangle, \ldots,\left|x_{n}\right\rangle\right\}$. The enterprise thinks that the probability of the university's preference for $y_{j}$ is obtained by projecting the initial state vector $|S\rangle$ onto the square of the length of the basic vector $\left|y_{j}\right\rangle$. The projection equation is $\left\langle y_{j} \mid S\right\rangle \cdot\left|y_{j}\right\rangle$, and the inner product of $\left|y_{j}\right\rangle$ and $|S\rangle$ is obtained by matrix formula (11). The probability of event $U y_{j}$ is $p\left(F y_{j}\right)=\left|\left\langle y_{j} \mid S\right\rangle\right|^{2}$.

$$
\left\langle y_{j} \| S\right\rangle=\sum_{k=1}^{n}\left(\Psi_{1} \cdot\left\langle y_{j} \mid x_{k}\right\rangle\right) .
$$

Firstly, considering that the university has a clear preference for innovation cooperation projects, the probability $p\left(E x_{i}\right)$ of enterprise choosing $x_{i}$ innovation cooperation projects is to project the state $\left|y_{j}\right\rangle$ to the $\left|x_{i}\right\rangle$ and then square it. The projection equation is $\left\langle x_{i} \mid y_{j}\right\rangle \cdot\left|y_{j}\right\rangle$. The inner product between $\left|x_{i}\right\rangle$ and $\left|y_{j}\right\rangle$ is $\left\langle x_{i} \mid y_{j}\right\rangle=\left\langle y_{j}|| x_{i}\right\rangle$.

So the probability of event $E x_{i}$ is $p\left(E x_{i}\right)\left|\left\langle x_{i} \mid y_{j}\right\rangle\right|^{2}$.

The probability of path $|S\rangle \longrightarrow\left|y_{j}\right\rangle \longrightarrow\left|x_{i}\right\rangle$ $p\left(U y_{j}\right) p\left(E x_{i}\right)=\left|\left\langle y_{j} \mid S\right\rangle\right|^{2} \cdot\left|\left\langle x_{i} \mid y_{j}\right\rangle\right|^{2}$.

Similarly, the probability of path $|S\rangle \longrightarrow\left|y_{j}\right\rangle \longrightarrow\left|x_{i}\right\rangle$ is $\left|\left\langle x_{i} \mid S\right\rangle\right|^{2} \cdot\left|\left\langle y_{j} \mid x_{i}\right\rangle\right|^{2}$. The probability of path $|S\rangle \longrightarrow\left|y_{j}\right\rangle \longrightarrow\left|x_{i}\right\rangle$ is not equal to that of the opposite path $|S\rangle \longrightarrow\left|x_{i}\right\rangle \longrightarrow\left|y_{j}\right\rangle$.

The sequence in which decision makers consider the opinions of university-enterprise collaborative members will affect their preference. The relationship between the size of $\left|\left\langle y_{j} \mid S\right\rangle\right|^{2}$ and $\left|\left\langle x_{i} \mid S\right\rangle\right|^{2}$, i.e., the size of the initial preference $\varphi_{j}$ of the university for project $y_{j}$ and the size of the initial preference $\Psi_{i}$ of enterprise for project $x_{i}$, determines the influence of the sequence of consideration.

When $\left|\varphi_{j}\right|>\left|\Psi_{i}\right|$, the enterprise considers their own preference before university preference. The probability for enterprise choosing innovation cooperation project $\varphi$ will be reduced, and the preference selection probability of enterprises will concentrate on their own preference state. The enterprise preference selection probability will increase the probability of choosing innovation cooperation project $i$ when the enterprise considers university's preference first and then their own preference state. The preference of the university will enable the enterprise to make clearer their own choice preference.

When $\left|\varphi_{j}\right|<\left|\Psi_{i}\right|$, there will be an opposite change in preference probability.

2.3.2. Decision-Making in the Complex Mixed State. The collaborative decision-making of universities and enterprises with sequential effect can be based on the results of exhaustive and mutually exclusive events of all known innovation projects of enterprises and universities. These events cannot be decomposed or improved into more specific results; instead, they are a complete measurement in quantum theory. In addition, the sequential effect can also be verified in the case of rough measurement. When the enterprise or the university can only identify preference from $x_{1}, x_{2}, \ldots, x_{n}$, the decision-making between universities and enterprises is based on rough measurement in quantum theory, and the selection event can be split.

For example, innovation projects can be divided into risk preference type and risk aversion type. For the consideration of university-enterprise collaborative innovation, the university-enterprise collaborative innovation team intends to choose between risk preference innovation cooperation project $x_{1}^{\prime}, x_{2}^{\prime}, \ldots, x_{k}^{\prime}$ and risk aversion innovation cooperation project $x_{1}^{\prime \prime}, x_{2}^{\prime \prime}, \ldots, x_{h}^{\prime \prime}$. Among them, $k+h=n$, and the corresponding expression based on the perspective of the university is risk preference innovation cooperation project $y_{1}^{\prime}, y_{2}^{\prime}, \ldots, y_{k}^{\prime}$ and risk aversion innovation cooperation project $y_{1}^{\prime \prime}, y_{2}^{\prime \prime}, \ldots, y_{h}^{\prime \prime}$. When the risk preference of innovation cooperation project is different between university and enterprise, the event can be decomposed from pure state to definite state and from mixed state to pure state.

The following two kinds of preference judgment sequence are taken as an example. The enterprise wants to choose a risk preference innovation project when they think that the university wants to choose a risk averse innovation project; the enterprise wants to choose a risk averse innovation project when they think that the university wants to choose a risk preference innovation project.

(1) Pure State Transition. The university has clearly informed the enterprise of wanting to choose risk averse innovation cooperation project $y_{a}^{\prime \prime}$ and needing to calculate the probability of the enterprise choosing a risk preference type cooperation project from risk preference projects and risk aversion innovation cooperation projects. $\left|x_{1}^{\prime}\right\rangle,\left|x_{2}^{\prime}\right\rangle, \ldots,\left|x_{k}^{\prime}\right\rangle$ is orthogonal, so the enterprise can choose one of $k$ innovation cooperation projects and need to calculate the probability of events crossing $\left\{\left|x_{1}^{\prime}\right\rangle \cup\left|x_{2}^{\prime}\right\rangle \cup \cdots \cup\left|x_{k}^{\prime}\right\rangle\right\}$. According to the quantum model, the original state $|S\rangle$ is modified to the modified state $\left|S_{R}\right\rangle$ by the basis vector $\left|x_{i}^{\prime \prime}\right\rangle$.

In quantum theory, $\left|y_{a}^{\prime \prime}\right\rangle$ is called pure state, which is the basis vector in the basic coordinate system 
$\left\{y_{1}^{\prime}, y_{2}^{\prime}, \ldots, y_{k}^{\prime}, y_{1}^{\prime \prime}, y_{2}^{\prime \prime}, \ldots, y_{h}^{\prime \prime}\right\}$, and it is also the superposition state in the basic coordinate system $\left\{x_{1}^{\prime}, x_{2}^{\prime}, \ldots, x_{k}^{\prime}, x_{1}^{\prime \prime}, x_{2}^{\prime \prime}, \ldots, x_{h}^{\prime \prime}\right\}$. Probability P1: first the risk aversion innovation cooperation project $y_{a}^{\prime \prime}$ is considered, and the risk preference project, which is equal to the square length of $\left|y_{a}^{\prime \prime}\right\rangle$ projected on the plane crossed by $\left\{\left|x_{1}^{\prime}\right\rangle \cup\left|x_{2}^{\prime}\right\rangle \cup \cdots \cup\left|x_{k}^{\prime}\right\rangle\right\}$, is then considered.

$$
\left.p_{1}=\|\left(\sum_{i=1}^{k}\left|x_{i}^{\prime}\right\rangle\left\langle x_{i}^{\prime}|| y_{a}^{\prime \prime}\right\rangle\right)\left\|^{2}=\sum_{i=1}^{k}\right\|\left\langle x_{i}^{\prime} \| y_{a}^{\prime \prime}\right\rangle\left|x_{i}^{\prime}\right\rangle\left\|^{2}=\sum_{i=1}^{2}\right\| x_{i}^{\prime}\right\rangle\left.\left|y_{a}^{\prime \prime}\right\rangle\right|^{2} .
$$

(2) Transition from Mixed State. After the enterprise has defined their preference state and wants a risk preference innovation project, they consider the probability that the university wants a risk averse innovation project, which discards the information of which risk preference innovation project is actually selected. In this case, before they judge whether the university wants risk averse innovation projects, their preference for the risk types of innovation cooperation projects has become clear, which leads to a mixed state. The probability of choosing $x_{b}^{\prime}$ from risk preference innovation projects is $\left|\left\langle x_{b}^{\prime} \mid S\right\rangle\right|^{2} / \sum_{i=1}^{k}\left|\left\langle x_{i}^{\prime} \mid S\right\rangle\right|^{2}$.

The enterprise thinks that the university wants to choose a risk averse innovation project $y_{a}^{\prime \prime}$, then the probability of enterprises choosing $x_{b}^{\prime}$ is $\left|\left\langle y_{a}^{\prime \prime}|| x_{b}^{\prime}\right\rangle\right|^{2}$. Therefore, the total probability $p_{2}$ of crossing $\left|y_{a}^{\prime \prime}\right\rangle$ after choosing a risk preference innovation project is equal to

$$
p_{2}=\sum_{i=1}^{k}\left(\frac{\left|\left\langle x_{i}^{\prime}|| S\right\rangle\right|^{2}}{\sum_{i=1}^{k}\left|\left\langle x_{i}^{\prime}|| S\right\rangle\right|^{2}} \cdot\left|\left\langle y_{a}^{\prime \prime} \mid x_{i}^{\prime}\right\rangle\right|^{2}\right) \text {. }
$$

In rough measurement, $p_{1}$ (enterprises choosing risk aversion innovation projects/universities choosing risk aversion innovation projects $y_{a}^{\prime \prime}$ ) is not equal to $p_{1}$ (university choosing risk aversion innovation projects $y_{a}^{\prime \prime} /$ enterprise choosing risk preference innovation projects).

2.3.3. Mutual Interference of University-Enterprise Preference Judgment. Two simple symbols $p(E)$ and $p(U)$ are used to mark enterprise preference and university preference, respectively. First the probability of event $E$ is observed, and then the change of probability is observed according to the sequence of events from $U$ to $E$. When the enterprise makes the choice according to the initial state, $p(E)=\| p_{E}|S\rangle \|^{2}$. According to the sequence of events from $U$ to $E$, that is, when the enterprise considers the preference of the university before their own preference, the probability of selection is $\| p_{E} p_{U}|S\rangle \|^{2}$. According to the quantum probability, the interference is $\operatorname{Int}_{E}=p(E)-p_{T}(E)$. In order to make a more detailed analysis, the probability of preference events is decomposed as follows:

$$
\begin{aligned}
p(E) & =\| P_{E}|S\rangle\left\|^{2}=\right\| P_{E} I|S\rangle\left\|^{2}=\right\| P_{E}\left(P_{U}+P_{\bar{U}}\right)|S\rangle \|^{2}, \\
& =\| P_{E} P_{U}|S\rangle\left\|^{2}+\right\| P_{E} P_{\bar{U}}|S\rangle \|^{2}+\left[\left\langle S\left|P_{U} P_{E} P_{\bar{U}}\right| S\right\rangle\left|+\left\langle S\left|P_{\bar{U}} P_{E} P_{U}\right| S\right\rangle\right|\right] \\
& =p_{T}(E)+\operatorname{Int}_{E} .
\end{aligned}
$$

If $P_{U} P_{E}=P_{E} P_{U}$, all the projections from the initial state to the base vector are interchangeable. Only when the projectors are not interconnected can interference occur. It may be positive or negative; thus, the law of total probability is violated. An important feature of quantum theory can be expressed by complex numbers, which can be better decomposed to express interference effect:

$$
\begin{aligned}
\text { Int }_{E} & =\left\langle S\left|P_{U} P_{E} P_{\bar{U}}\right| S\right\rangle\left|+\left\langle S\left|P_{\bar{U}} P_{E} P_{U}\right| S\right\rangle\right| \\
& =\left\langle S\left|P_{\bar{U}} P_{E} P_{U}\right| S\right\rangle\left|+\left\langle S\left|P_{\bar{U}} P_{E} P_{U}\right| S\right\rangle\right| * \\
& =2 \cdot\left\langle S\left|P_{\bar{U}} P_{E} P_{U}\right| S\right\rangle \mid \cdot \cos (\theta) .
\end{aligned}
$$

The inner product $\left\langle S\left|P_{\bar{U}} P_{E} P_{U}\right| S\right\rangle$ in general forms is a complex number, where $\theta$ is the phase angle of the inner product $\left\langle S\left|P_{\bar{U}} P_{E} P_{U}\right| S\right\rangle$. When $\left\langle S\left|P_{\bar{U}} P_{E} P_{U}\right| S\right\rangle=0$, that is, the event projection between the enterprise's cognitive state and the university's cognitive state can be communicated, the interference effect is zero. In addition, when $\cos (\theta)=0$, the interference is zero. When the imaginary part of the complex number is zero, the complex number can be a real number. When $\cos (\theta)=1$, the interference is the most positive, and when $\cos (\theta)=-1$, the interference is the most negative.

When the path similarity is within $|\pi / 2|$, the interference effect is positive, and the smaller is the value $|\theta|$, the greater is the positive interference effect. When the path similarity is between $|\pi / 2|$ and $|\pi|$, the interference effect is negative, and the greater is the value $|\theta|$, the greater is the negative interference effect.

According to the different phases obtained by different path wave functions involved in quantum mechanics, it is considered that the phase angles between different sequential paths are also different in university-enterprise collaborative decision-making. This interference effect is an abstract understanding of interference effect regarding different path effects.

2.4. Examples. Firstly, the evaluation data of universities and enterprises on four cooperative innovation projects are collected, and then the evaluation data are normalized in turn. 
As shown in Table 1, there are significant differences in the evaluation of cooperative innovation projects between universities and enterprises.

2.4.1. Decision Analysis of Independent Innovation of Universities or Enterprises. Universities or enterprises do not consider each other's preferences when making decisions independently. The probability of enterprise innovation decision is projected to the $X_{1}$ axis through the state vector $|S\rangle$, and the transition amplitude is $\left|X_{1}\right\rangle\left\langle X_{1} \mid S\right\rangle=0.520 \cdot\left|X_{1}\right\rangle$, the square of the amplitude, $\| 0.520 \cdot\left|X_{1}\right\rangle\left\|^{2}=|0.520|^{2} \cdot\right\|\left|X_{1}\right\rangle \|^{2}=(0.270) \cdot 1$.

The amplitude and amplitude square of the state vector projected to other axes can be obtained in the same way. Therefore, the probability of the four projects of the decision maker of innovation project generated by the enterprise from their own will is $0.270,0.144,0.032,0.553$, and the sum of probability is 1 . The probability of the four projects of the independent decision maker of innovation project produced by the university is $0.348,0.084,0.152$, and 0.416 , and the sum of probability is 1 .

Suppose that the first and second of the four projects are risk preference innovation projects. Then, the event with which the enterprise chooses risk preference innovation projects can be expressed as "project 1, project 2," which is represented by spanned two-dimensional subspace: $\left\{\left|X_{1}\right\rangle,\left|X_{2}\right\rangle\right\}$ in the multidimensional space of quantum cognition. The probability of this event is obtained by projecting the state vector $|S\rangle$ into this subspace. The projection equation is as follows:

$$
\begin{aligned}
& \left(\left|X_{1}\right\rangle\left\langle X_{1}|+| X_{2}\right\rangle\left\langle X_{2}\right|\right)|S\rangle \\
& =\left|X_{1}\right\rangle\left\langle X_{1} \mid S\right\rangle+\left|X_{2}\right\rangle\left\langle X_{2} \mid S\right\rangle \\
& =0.520 \cdot\left|X_{1}\right\rangle+0.380 \cdot\left|X_{2}\right\rangle .
\end{aligned}
$$

The probability of this event is the square of the length of the projection equation of this event: $\| 0.520 \cdot\left|X_{1}\right\rangle+0.380 \cdot\left|X_{2}\right\rangle \|^{2}=0.414$.

The probability of the enterprise choosing risk preference innovation projects is 0.414 . Similarly, the probability of the university choosing risk preference innovation projects is 0.432 .

2.4.2. Innovation Joint Decision Analysis. Based on the method given above, the innovation joint decision probability matrix is constructed as follows:

$$
\left[\begin{array}{llll}
0.307 & 0.224 & 0.106 & 0.439 \\
0.089 & 0.065 & 0.031 & 0.127 \\
0.035 & 0.025 & 0.012 & 0.050 \\
0.022 & 0.016 & 0.008 & 0.032
\end{array}\right] \text {. }
$$

Based on the innovation joint decision probability matrix, the unitary matrix $U_{E U}$ satisfying the conditions is constructed.

$$
U_{E U}=\left[\begin{array}{llll}
0.495 & 0.362 & 0.171 & 0.708 \\
0.144 & 0.105 & 0.050 & 0.205 \\
0.056 & 0.041 & 0.019 & 0.080 \\
0.036 & 0.026 & 0.012 & 0.052
\end{array}\right] .
$$

The transformation matrix $U_{U E}$ is as follows:

$$
U_{U E}=\left[\begin{array}{llll}
0.495 & 0.144 & 0.056 & 0.036 \\
0.362 & 0.105 & 0.041 & 0.026 \\
0.171 & 0.050 & 0.019 & 0.012 \\
0.708 & 0.205 & 0.080 & 0.052
\end{array}\right] .
$$

The university preferences that the enterprise thinks are as follows:

$$
\left[\begin{array}{l}
0.907 \\
0.076 \\
0.012 \\
0.005
\end{array}\right]
$$

It can be seen that there is a significant difference between the preference of the university that the enterprise thinks and the preference of the university itself. The university thinks that the preference of the enterprise is as follows:

$$
\left[\begin{array}{l}
0.270 \\
0.144 \\
0.032 \\
0.553
\end{array}\right]
$$

It can be seen that the preference of the enterprise perceived by the university is similar to that of the enterprise itself. The example in this paper fully shows the value of quantum probability in the evaluation of cooperative projects. In this paper, quantum cognitive theory is used to reveal the superposition effect in cooperative decisionmaking. It subverts the situation where the classical decision theory is used to assign different probability and utility to each result. In the quantum model constructed in this paper, difference does not exist in every pair of superposition effect and single effect, which indicates that quantum probability is more suitable to explain the decision-making mechanism of synergistic effect.

\section{Results and Discussion}

The internal mechanism of university-enterprise collaborative innovation decision-making is based on the quantum attribute of university-enterprise collaborative innovation decision-making. The uncertainty of quantum theory is reflected in the vague preference state of decision makers; the formation of decision-making is related to the degree to which the decision maker receives the opinions of university-enterprise collaborative innovation members, and different opinions and suggestions form the entanglement and 
TABLE 1: Evaluation data of innovation projects by universities and enterprises.

\begin{tabular}{lcc}
\hline Project & Enterprises & Universities \\
\hline 1 & 0.520 & 0.590 \\
2 & 0.380 & 0.290 \\
3 & 0.180 & 0.390 \\
4 & 0.744 & 0.645 \\
\hline
\end{tabular}

superposition attributes in quantum theory. The order of decision makers' evaluation of recognition information produces the sequential effect that can be explained by quantum theory; the preference of university-enterprise is related to the degree of decision maker's receiving opinions from university-enterprise collaborative innovation members. Binary variable decision-making produces the interpretable interference effect in quantum theory. Compared with the traditional method based on the hypothesis of "rational man" which excludes uncertainty and entanglement, quantum method can explain the change of decision maker's cognitive state in the most direct way.

University-enterprise collaborative innovation decisionmaking tends to be an incompatible representation in quantum cognition. When considering the cognitive preference of the university and the enterprise, there will be two kinds of variables, so it is necessary to explore whether the two groups of variables are compatible. Whether the two variables are compatible or not depends on whether the decision maker has enough knowledge and experience to form a compatible representation. The two variables in the collaborative innovation decision-making mode of university-enterprise are more consistent with incompatible representation.

The opinions of university-enterprise collaborative innovation members will produce sequential effect and interference effect on decision makers of university-enterprise collaborative innovation. The interference effect and sequential effect of a single path are affected by the phase angle of the path wave and have different effects. When the total interference effect and sequential effect of the decision result are fully considered, the different sequence will produce interpretable interference effect. When the final decision maker's personal preference is evaluated at last, the decisionmaking result can better reflect the process of mutual interference and compromise between the two preferences of the university and the enterprise, which tends to be the joint decision-making of university-enterprise collaborative innovation. Finally, when the decision maker first evaluates their own preference, the decision-making result will be more inclined to the more explicit preference of the decision maker.

\section{Conclusion}

The research, as an analysis of the internal interaction mechanism, has analyzed the impact of university-enterprise on decision-making in collaborative innovation decisionmaking, which has certain restrictions on guiding enterprises to carry out innovation activities, and further research is needed in specific practice.
The model research only uses the geometric characteristics of Hilbert space in quantum theory to express the state of time and does not involve the quantum properties that can be calculated by Schrodinger equation, so it has some limitations in explaining the complex dynamic changes.

Considering the influence of external stakeholders in collaborative innovation decision-making between the university and the enterprise, this cognitive model can be extended to more dimensions of incompatible representation, and quantum analysis may involve the process of quantum dynamic random walk. In the field of collaborative innovation, how to make good use of the sequential effect and interference effect of individual decision-making and university-enterprise collaborative innovation decisionmaking is worthy of in-depth study. In the future research, researchers can try this method in the fields of portfolio [30], product development [33], supply chain management [34], and industrial competition analysis [35].

\section{Data Availability}

The experimental data used to support the findings of this study are included within the article.

\section{Conflicts of Interest}

The authors declare that they have no conflicts of interest.

\section{Acknowledgments}

This work was supported by the National Social Science Foundation of China (no. 20BGL203).

\section{References}

[1] J. Eisert, M. Wilkens, and M. Lewenstein, "Quantum games and quantum strategies," Physical Review Letters, vol. 83, no. 15 , pp. 3077-3080, 1999.

[2] L. A. Cotfas, "A finite-dimensional quantum model for the stock market," Physica A: Statistical Mechanics \& Its Applications, vol. 392, no. 2, pp. 371-380, 2012.

[3] A. Ataullah, I. Davidson, and M. Tippett, "A wave function for stock market returns," Physica A: Statistical Mechanics and Its Applications, vol. 388, no. 4, pp. 455-461, 2009.

[4] F. Bagarello, "A quantum statistical approach to simplified stock markets," Physica A: Statistical Mechanics and Its Applications, vol. 388, no. 20, pp. 4397-4406, 2009.

[5] F. Bagarello, "Stock markets and quantum dynamics: a second quantized description," Physica A: Statistical Mechanics and Its Applications, vol. 386, no. 1, pp. 283-302, 2009.

[6] J. R. Busemeyer and Z. Wang, "What is quantum cognition, and how is it applied to psychology?" Current Directions in Psychological Science, vol. 24, no. 3, pp. 163-169, 2015.

[7] P. Bruza, J. R. Busemeyer, and L. Gabora, "Introduction to the special issue on quantum cognition," Journal of Mathematical Psychology, vol. 53, no. 5, pp. 303-305, 2009.

[8] J. R. Busemeyer, Z. Wang, and J. T. Townsend, "Quantum dynamics of human decision-making," Journal of Mathematical Psychology, vol. 50, no. 3, pp. 220-241, 2006.

[9] E. M. Pothos and J. R. Busemeyer, "A quantum probability explanation for violations of 'rational' decision theory," 
Proceedings of the Royal Society B: Biological Sciences, vol. 276, no. 1665, pp. 2171-2178, 2009.

[10] V. I. Yukalov and D. Sornette, "Decision theory with prospect interference and entanglement," Theory and Decision, vol. 70, no. 3, pp. 283-328, 2011.

[11] E. M. Pothos and J. R. Busemeyer, "Can quantum probability provide a new direction for cognitive modeling?" Behavioral and Brain Sciences, vol. 36, no. 3, pp. 255-274, 2013.

[12] T. Filk and A. V. Muller, "Quantum physics and consciousness: the quest for a common conceptual foundation," Mind and Matter, vol. 7, no. 1, pp. 59-79, 2009.

[13] A. L. Mogiliansky, S. Zamir, and H. Zwirn, "Type indeterminacy: a model of the KT (Kahneman-Tversky)-man," Journal of Mathematical Psychology, vol. 53, no. 5, pp. 349361, 2003.

[14] Z. Wang, T. Solloway, R. M. Shiffrin, and J. R. Busemeyer, "Context effects produced by question orders reveal quantum nature of human judgments," Proceedings of the National Academy of Sciences, vol. 111, no. 26, pp. 9431-9436, 2014.

[15] G. Niestegge, "An approach to quantum mechanics via conditional probabilities," Foundations of Physics, vol. 38, no. 3, pp. 241-256, 2008.

[16] D. Aerts and S. Sozzo, "Quantum structure in cognition: why and how concepts are entangled," Quantum Interaction, vol. 7052, no. 5, pp. 116-127, 2011.

[17] P. Beim Graben, T. Filk, and H. Atmanspacher, "Epistemic entanglement due to non-generating partitions of classical dynamical systems," International Journal of Theoretical Physics, vol. 52, no. 3, pp. 723-734, 2013.

[18] J. M. Yearsley and J. R. Busemeyer, "Quantum cognition and decision theories: a tutorial," Journal of Mathematical Psychology, vol. 74, pp. 99-116, 2015.

[19] D. W. Moore, "Measuring new types of question-order effects," Public Opinion Quarterly, vol. 66, no. 1, pp. 80-91, 2002.

[20] D. Aerts, S. Sozzo, and T. Veloz, "Quantum structure in cognition and the foundations of human reasoning," International Journal of Theoretical Physics, vol. 54, no. 12, pp. 4557-4569, 2015.

[21] J. R. Busemeyer and Z. Wang, "Quantum cognition: key issues and discussion," Topics in Cognitive Science, vol. 6, no. 1, pp. 43-46, 2014.

[22] P. D. Kvam, T. J. Pleskac, S. Yu, and J. R. Busemeyer, "Interference effects of choice on confidence: quantum characteristics of evidence accumulation," Proceedings of the National Academy of Sciences, vol. 112, no. 34, pp. 1064510650, 2015.

[23] J. R. Busemeyer, E. M. Pothos, R. Franco, and J. S. Trueblood, "A quantum theoretical explanation for probability judgment errors," Psychological Review, vol. 118, no. 2, pp. 193-218, 2011.

[24] D. Aerts and S. Sozzo, "Quantum structure in cognition, origins, developments, successes and expectations," Computer Science, vol. 11, no. 5, pp. 314-348, 2015.

[25] P. B. Graben and H. Atmanspacher, "Complementarity in classical dynamical systems," Foundations of Physics, vol. 36, no. 2, pp. 291-306, 2006.

[26] L. C. White, E. M. Pothos, and J. R. Busemeyer, "Insights from quantum cognitive models for organizational decision making," Journal of Applied Research in Memory and Cognition, vol. 4, no. 3, pp. 229-238, 2015.

[27] U. Lichtenthaler, "Open innovation: past research, current debates, and future directions," Academy of Management Perspectives, vol. 25, no. 1, pp. 75-93, 2011.
[28] H. Garriga, G. von Krogh, and S. Spaeth, "How constraints and knowledge impact open innovation," Strategic Management Journal, vol. 34, no. 9, pp. 1134-1144, 2013.

[29] S.-Y. Wang, W.-M. Chen, R. Wang, and X.-L. Wu, "Multiobjective evaluation of co-evolution among innovation populations based on Lotka-Volterra equilibrium," Discrete Dynamics in Nature and Society, vol. 2021, Article ID 5569108, 14 pages, 2021.

[30] Q. Wu, X. Liu, J. Qin, W. Wang, and L. Zhou, "A linguistic distribution behavioral multi-criteria group decision making model integrating extended generalized TODIM and quantum decision theory," Applied Soft Computing Journal, vol. 2020, Article ID 106757, 20 pages, 2020.

[31] Q. Wu, X. Liu, L. Zhou, Z. Tao, and J. Qin, “A quantum framework for modeling interference effects in linguistic distribution multiple criteria group decision making," IEEE Transactions on Systems, Man, and Cybernetics: Systems, vol. 2021, Article ID 3072388, 16 pages, 2021.

[32] J. R. Busemeyer and P. D. Bruza, Quantum Models of Cognition and Decision, Cambridge University Press, Cambridge, UK, 2012.

[33] S.-Y. Wang, W.-M. Chen, and Y. Liu, "Collaborative product portfolio design based on the approach of multi choice goal programming," Mathematical Problems in Engineering, vol. 2021, Article ID 6678533, 16 pages, 2021.

[34] Q. Wu, L. Zhou, Y. Chen, and H. Chen, "An integrated approach to green supplier selection based on the interval type-2 fuzzy best-worst and extended VIKOR methods," Information Sciences, vol. 502, pp. 394-417, 2019.

[35] S.-Y. Wang, W.-M. Chen, and X.-L. Wu, "Competition analysis on industry populations based on a three-dimensional Lotka-Volterra model," Discrete Dynamics in Nature and Society, vol. 2021, Article ID 9935127, 15 pages, 2021. 